\title{
Nephro-Protective Effect of Kangqianling Decoction on Chronic Renal Failure Rats
}

By: Liqun He, Peicheng Shen, Qiang Fu, Jun Li, Mo Dan, Xiaoyan Wang, and Wei Jia

He, L., Shen, P., Fu, Q., Li, J., Dan, M., Wang, X., \& Jia, W. (2009). Nephro-protective effect of Kangqianling decociton on chronic renal failure rats. Journal of Ethnopharmacology, 122, 367-373.

\author{
***Note: This version of the document is not the copy of record. Made available courtesy of \\ Elsevier. Link to Full Text: \\ http://www.sciencedirect.com/science/article/pii/S0378874108006946
}

\begin{abstract}
:
Ethnopharmacological relevance: Kangqianling decoction (KQL), the modified formulation of a classical Chinese prescription named Taohongsiwu decoction, was clinically employed to treat renal fibrosis in chronic renal failure.

Aim of the study: The present study was designed to examine whether KQL has a protective effect on renal function in association with transforming growth factor- $\beta$ (TGF- $\beta$ ), angiotensin II (Ang II), tumor necrosis factor- $\alpha$ (TNF- $\alpha$ ), nuclear factor- $\mathrm{KB}$ (NF- $\mathrm{\kappa B}$ ) in rats with $5 / 6$ renal ablation (Nx)-induced chronic renal failure.

Results: In renal function deterioration progression, the high expression of serum creatinine (Scr), 24-h urine protein and systolic blood pressure were markedly $(P<0.05$ or $P<0.01)$ restored by KQL, respectively, at 4 and 8 weeks. The increasing expressions of renal Ang II $(P<0.05)$, angiotensin II1-receptor (AT1R) $(P<0.05)$, TNF- $\alpha(P<0.05)$, NF-kB $(P<0.001)$ and urine TGF- $\beta 1(P<0.05)$ were reduced by the treatment of KQL. Immunohistochemical study further confirmed the nephro-protective activity of KQL as compared to the control and Sham group.

Conclusions: The results indicate that KQL is able to protect renal function via ameliorating experimental rat renal failure as found in these renal functional parameters.

\section{Article:}

INTRODUCTION

Some traditional Chinese herbal formulations documented in the ancient Chinese pharmacopoeia or monographs have been employed for chronic renal failure, especially at early and middle stage (He et al., 2003, Li et al., 2003, He and Cai, 2005a and He et al., 2005b). Kangqianling decoction (KQL), composed of radix salvia miltiorrhizae (Dansen), fructus jujubae (Dahuang), badix achyranthis bidentatae (Niuxi), and semen perwicae (Taoren), radix angelicae sinensis (danggui), was originated from one of the most famous Chinese prescription, Taohongsiwu decoction (compared with KQL, plus Paeonia, szechwan lovage rhizome flos carthami, and without fructus jujubae, badix achyranthis bidentatae), which has been widely used to treat hematomas (Jin et al., 2006), nephrotic syndrome (Guo, 2006) and chronic glomerulonephritis (Xia et al., 2007), etc. In clinical practice, KQL could significantly decrease serum creatinine (Scr) and blood urea nitrogen (BUN) as well as delayed progression of chronic renal failure.
\end{abstract}


KQL could significantly lower creatinine and urea nitrogen of early metaphase chronic renal failure (CKD2-3) patients, and were widely used in the treatment of early metaphase chronic renal failure (Chen and He, 2006).

Almost all progressive chronic renal failure is the consequence of destructive fibrosis, glomeruloscerosis and extracellular matrix hyperplasia. The fibrogenic signaling phase is characterized by the release of soluble factors that have fibrosis-promoting effects. Several growth factors and cytokines have been implicated with primary roles suggested for transforming growth factor- $\beta$ (TGF- $\beta$ ), angiotensin II (Ang II) and endothelin-1. Additional factors may participate including platelet-derived growth factor, basic fibroblast growth factor, tumor necrosis factor- $\alpha$ (TNF- $\alpha$ ) and interleukin-1 (IL-1) (Allison, 2000). Hypertension is usually associated with the development of vascular and renal fibrosis, which is characterized by structural changes in vasculature caused by increased synthesis and rearrangement of extracellular matrix proteins (Weistuch and Dworkin, 1992). Nuclear factor- $\mathrm{B}(\mathrm{NF}-\kappa \mathrm{B}), \mathrm{a}$ family of dimeric transcription factors that regulate the expression of numerous genes involved in inflammation and cell proliferation, has been found to be activated in experimental renal disease. Importantly, NF- $\kappa \mathrm{B}$ activation can be modulated by pharmacological maneuvers both in vivo and in vitro. Indeed, it is now widely acknowledged that the anti-inflammatory action of steroids is basically obtained through the inhibition of the transactivation of NF- $\kappa \mathrm{B}$-dependent genes. Additionally, some of the beneficial effects of angiotensin-converting enzyme inhibitors and statins may, at least in part, be mediated by an inhibition of NF- $\kappa$ B activation (Guijarro and Egido, 2001).

There is little experimental data regarding probable molecular mechanisms of nephro-protective action of KQL on chronic renal failure although being used clinically for decades. Therefore, in the present investigation, we examined the effects of KQL against 5/6 renal ablation (Nx)induced chronic renal failure. At the same time, we investigate the influence of renal mass reduction (RMR) caused by Nx on gene expression of Ang II, AT1R, NF- $\kappa B$, TNF- $\alpha$ and TGF$\beta 1$ and evaluate the correlation between expression of these genes and activity of the intrarenal rennin-angiotensin systems.

\section{MATERIALS AND METHODS \\ Animals}

Munich-Wistar rats (200 \pm 20 g) (BiKai, Shanghai, China) were used for the study. All the rats were individually housed in stainless steel wire-mesh cages, and fed ad libitum with standard solid chow (BiKai Animal Lab. Company, Shanghai, China) containing 24.5\% protein. Room temperature and humidity were regulated at $24 \pm 1{ }^{\circ} \mathrm{C}$ and $45 \pm 15 \%$, respectively. All the procedures were in strict accordance with the PR China legislation on the use and care of laboratory animals and with the guidelines established by the Institute for Experimental Animals of Shanghai Jiao Tong University and were approved by the University Committee for animal experiments.

\section{Preparation of Kangqianling decoction}

KQL is composed of radix salvia miltiorrhizae (from province of Sichuan) $30 \mathrm{~g}$, fructus jujubae (from province of Gansu) $9 \mathrm{~g}$, badix achyranthis bidentatae (from province of Henan) $15 \mathrm{~g}$, and semen perwicae (from province of Hebei) $12 \mathrm{~g}$, radix angelicae sinensis (from province of 
Gansu) 15 g. All the herbal drugs were purchased from Shanghai Huayu Pharmaceutical Co., and identified by authority. The air-dried herbs were powdered and subjected to hot water extraction for $3 \mathrm{~h}$. The extract was concentrated, precipitated with 4 times alcohol, free of the solvent and then finally dispersed and dissolved in hot water and then cooled for rat administration. Final yield of prepared decoction was above $35 \%$.

\section{Experimental procedure}

Animals were divided into three groups: Sham Group (normal Sham-control), Control Group (empty vehicle-treated control), Treatment Group (received KQL), each group containing 12 animals. According to literatures with little modification (Ng et al., 1998 and Goncalves et al., 2004), at first, the left kidneys of 24 Munich-Wistar rats were 2/3 renal ablated. After 1 week, the right kidneys were ablated. These procedures were performed under anaesthesia with sodium pentobarbital (Shanghai Pharmaceutical Factory, Shanghai, China). Two weeks after Nx, the 24 rats were divided into 12 pairs, where two rats in each pair exhibited almost the same levels of Scr, BUN (Table 1). Then one rat of each pair were collected to be Treatment Group received KQL ( $n=12)$, at a daily dose of $0.75 \mathrm{~g}$ (crude herbal drugs)/100 g (body weight), converted according to clinical human dosage, orally for 8 weeks. The others were allocated to be Control Group (vehicle control, $n=12$ ) received empty vehicle parallel. For Sham Group, rats underwent a Sham operation consisting of laparotomy and manipulation of the renal pedicles but without damage to the kidney $(n=12)$.

Table 1: Effect of KQL on serum creatinine (Scr), blood urea nitrogen (BUN) and 24-h urinary protein excretion.

\begin{tabular}{|c|c|c|c|c|c|c|c|c|}
\hline \multirow[t]{2}{*}{ Group } & \multirow[t]{2}{*}{$N$} & \multicolumn{3}{|l|}{ BUN (mmol/L) } & \multicolumn{3}{|l|}{$\operatorname{Scr}(\mu \mathrm{mol} / \mathrm{L})$} & \multirow{2}{*}{$\begin{array}{l}24 \text { h urine protein (mg) } \\
\text { Eighth week }\end{array}$} \\
\hline & & Before treatment & Fourth week & Eighth week & Before treatment & Fourth week & Eighth week & \\
\hline Sham & 12 & $7.51 \pm 0.75$ & $6.79 \pm 0.70$ & $9.31 \pm 1.05$ & $19.00 \pm 4.00$ & $26.25 \pm 1.04$ & $18.88 \pm 1.55$ & $22.34 \pm 4.4$ \\
\hline Control & 12 & $16.17 \pm 0.99^{*}$ & $12.09 \pm 3.37$ & $14.85 \pm 2.83$ & $49.50 \pm 6.53^{*}$ & $50.56 \pm 15.83$ & $53.38 \pm 12.05$ & $41.47 \pm 8.07$ \\
\hline Treatment & 12 & $16.18 \pm 2.42^{*}$ & $9.81 \pm 2.93$ & $13.62 \pm 2.81$ & $49.23 \pm 9.36^{*}$ & $38.83 \pm 12.00^{\#}$ & $41.00 \pm 10.51^{\# \#}$ & $29.14 \pm 5.68^{\# \#}$ \\
\hline
\end{tabular}

Values are means \pm S.E.M.

$* P<0.05$, compared with Sham Group

$\# P<0.05$, compared with Control Group.

$\# \# P<0.01$, compared with Control Group.

Blood pressure was measured before treatment and every 2 weeks after the surgery. The levels of Scr, BUN, 24 h urine protein excretion and urine TGF- $\beta$ were determined at 4 or 8 weeks after starting the administration of KQL, respectively. On the eighth week, the animals were sacrificed and the remnant kidneys were collected for histopathological and gene expression studies.

Assessment of renal function and blood pressure. Scr and BUN was measured using a Beckman Cx4 analyser (Fullerton, CA, USA), respectively. 24 h urinary protein concentrations were determined by the Bradford method, adapted to a microtiter plate assay. Coomassie reagent (USB, Cleveland, $\mathrm{OH}$ ) was added to the diluted urine samples. After $10 \mathrm{~min}$, the absorbance at 595-nm wavelength was read on ELX800 microplate reader (Bio-Tek Instruments, VT). The protein concentrations were calculated by reference to bovine serum albumin (Sigma) standards. Systolic blood pressure was recorded by tail plethysmography using the BP2000 blood pressure analysis system (Visitech Systems, Inc., Apex, NC) in conscious rats at baseline and every 2 weeks throughout the experimental time course.

Assessment of immunohistochemistry. Immunostaining of NF- $\kappa \mathrm{B}$ in renal tissue sections was performed using the streptavidin-biotinylated peroxidase complex (SABC) method (Muller et 
al., 2000). The immunostaining of NF- $\mathrm{KB}$ was quantified using an image analyser IMS (FUDAN University of Medical Science Portrait Examination Center, Shanghai, China) by evaluating the positively stained area of the sections under the same light intensity for microscopy.

Real-time quantitative polymerase chain reaction (PCR) for TNF- $\alpha$, Ang II and AT1R. To investigate the mRNA expression of TNF- $\alpha$, Ang II and angiotensin II1-receptor (AT1R), realtime PCR (BC Living Creature Technique Co., Shanghai, China) was performed on the Opticon machine (FX Scientific Research Inc., Shanghai, China) ( [Karkar et al., 1992], [Ruiz-Ortega and Egido, 1997], [Wolf et al., 1999] and [Goncalves et al., 2004]). Briefly, total RNA was extracted from renal tissues and cultured cells by homogenization in TRIzol (Trizol box, from GIBCO BRC Inc., Shanghai, China). All of the RNA samples were treated with the RNase-free DNase I (GIBCO BRC Inc., Shanghai, China) before the RT-PCR. Real-time quantitative one-step RTPCR assay was performed to quantify mRNA using real-time PCR machine (FX Scientific Research Inc., Shanghai, China). The primers used for real-time RT-PCR were as follows:

TNF- $\alpha$ : forward 5'-CTCATTCCCGCTCGTGG-3'; reverse 3'CGTTTGGTGGTTCGTCTCC-5';

AT1R: forward 5'-CTTGTTCCCTTTCCTTATC-3'; reverse 3'-ACTCCACCTCAC TGTCCA-5';

Ang II: forward 5'-ACCTG CATGA GTGTT GATAGG-3'; reverse 3'-ACTTCA ATATC GTCAGTAACTGGAC-5'.

Quantitative analyses of TNF- $\alpha$, Ang II and AT1R expressions were performed using a quantitative image analysis system FR-2000 (FR Science and Technology Inc., Shanghai, China).

Characterization of monoclonal anti-TGF- $\beta$ antibody. The reactivity of the produced monoclonal antibodies with urine TGF- $\beta$ was screened by enzyme-linked immunosorbent assay (ELISA) using kit produced by Section Living Creature Technique Limited Company of Hangzhou, China (NO. 13409007) (Houlihan et al., 2002).

\section{Statistical analysis}

Results were expressed as the means \pm S.E.M. Statistical analyses were performed using GraphPad Prism 3.0 (GraphPad Software, Inc., San Diego, CA). Differences in blood pressure, Scr, BUN, 24 h urine protein and Urine TGF- $\beta$ at different time points (weeks $0-8$ ) among the groups, and differences of Ang II and AT1R activation, TNF- $\alpha$ expression and NF- $\kappa b$ accumulation in Sham Group, Control Group, Treatment Group were assessed by one-way analysis of variance (AOWAV) and $t$-test to judge whether the results were statistically significant. The critical $p$-value of both tests was set to 0.05 in this study.

RESULT

Effects of KQL on physiological biochemical parameters of NX rats 
Renal and systemic parameters were obtained at 0,4 and 8 weeks after Nx given (Table 1) (Fig. 1, Fig. 2, Fig. 3, Fig. 4 and Fig. 5). In Control Group, body weights were statistically different from those observed before treatment. Average food intake was similar among groups. There was significant change in body weight between the control Nx rats and treated Nx rats, although they were pair-fed. The body weights of KQL treated rats were significantly higher than empty vehicle treated ones. Even 4 weeks after Nx, the levels of Scr and BUN of Nx rats were markedly increased as compared with Sham rats. In renal function deterioration progression, the high expression of Scr and BUN were observed in the end of experimental research, while Scr was markedly restored by KQL, respectively, at both 4 and 8 weeks. Urinary protein excretion was also suppressed obviously at eighth week as compared with Nx rats in Control Group.
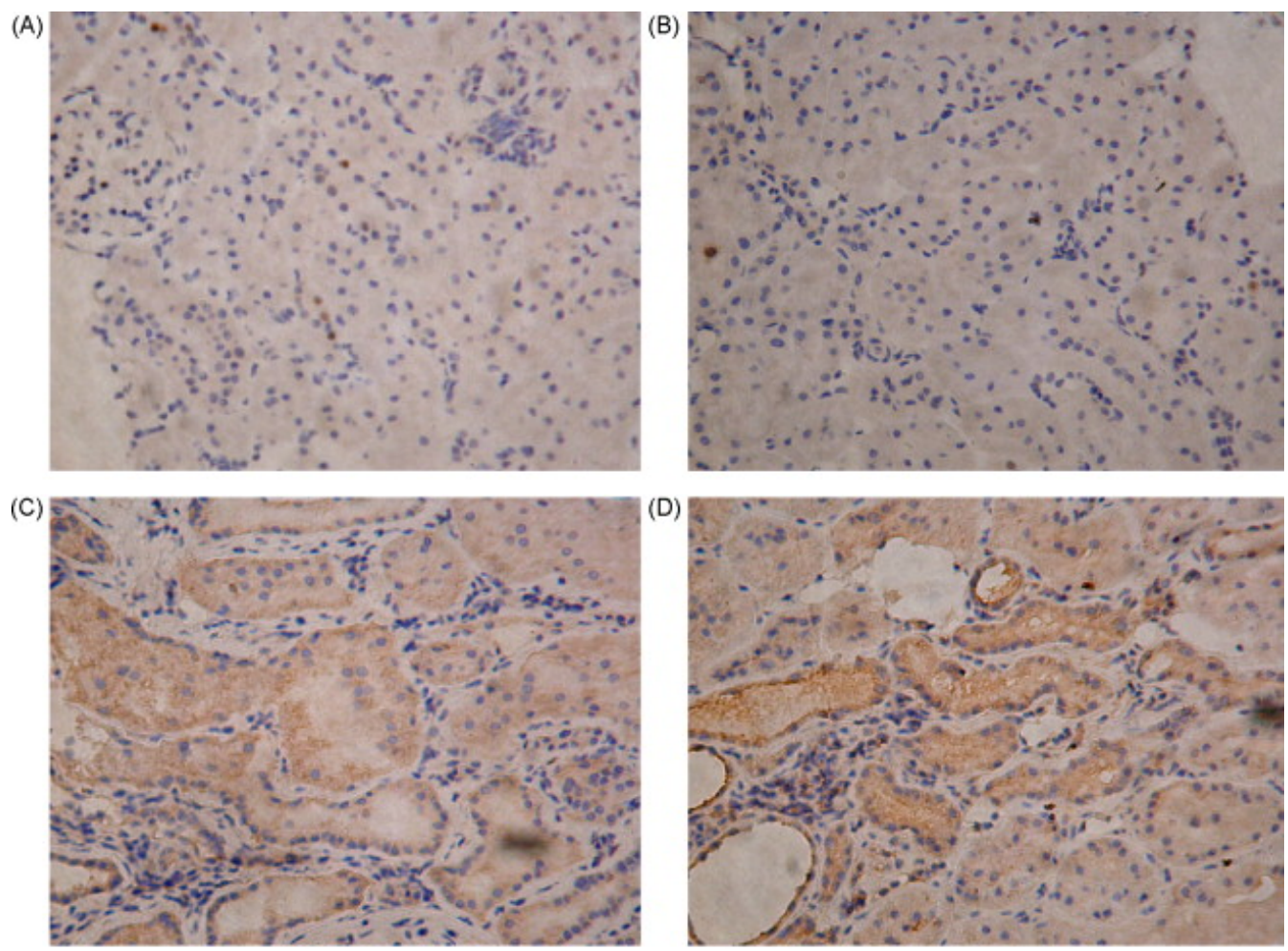

(E)
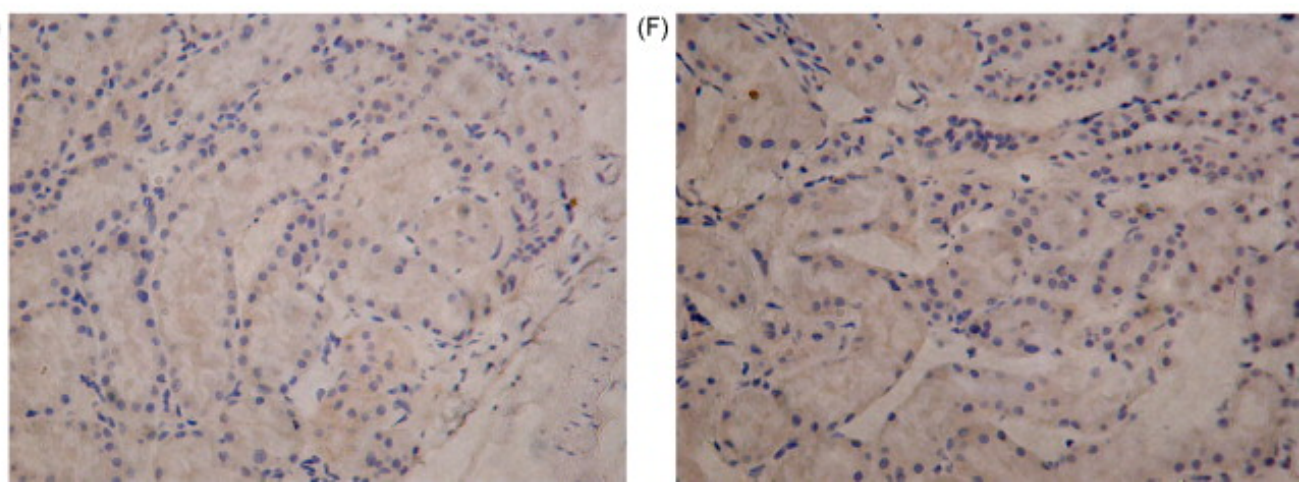

Figure 1: Effect of KQL on inhibiting renal NF- $\mathrm{KB}$ accumulation within the kidney in immunohistochemistry results. The accumulation of NF- $\mathrm{kB}$ in the glomerular and tubulointerstitium is demonstrated from Sham rats (A and B), Control rats (C and D), and Treatment rats (E and F) (original magnifications 100×). 


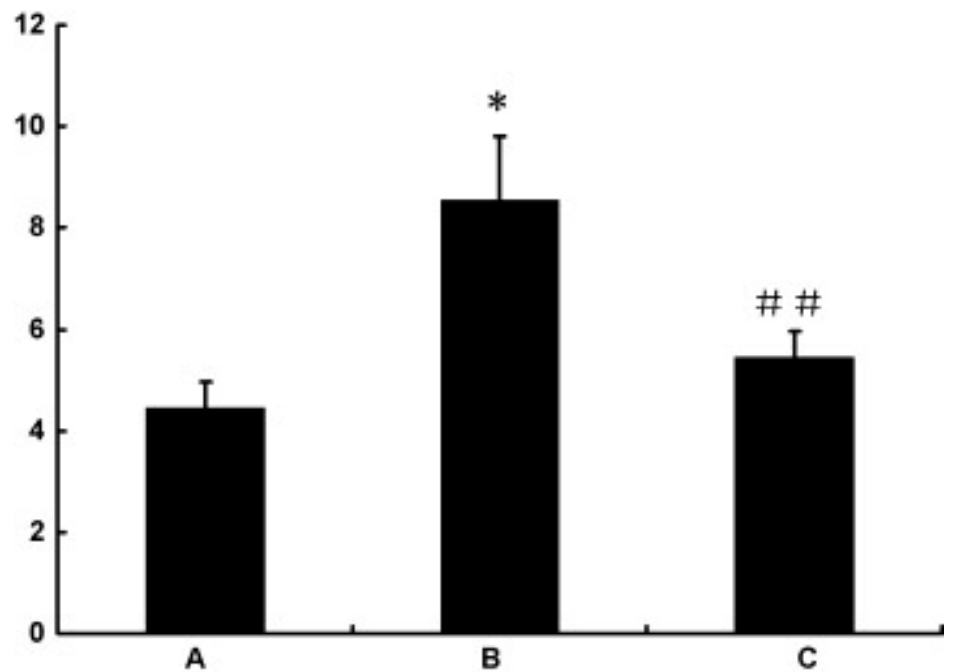

Figure 2: Semiquantitative analysis of the therapeutic effect of KQL on NF- $\mathrm{B}$ localization in the glomerulus and tubulointerstitium using the Quantitative Image System. Percentage of glomerular and tubulointerstitial NF- $\kappa \mathrm{B}$ deposition in Sham Group (A), Control Group (B) and Treatment Group (C). Each value represents mean \pm S.E.M. ${ }^{\#} P<0.05,{ }^{\# \#} P<0.001$ compared with Control Group; ${ }^{*} P<0.05, * * P<0.01$, compared with Sham Group.

(a)

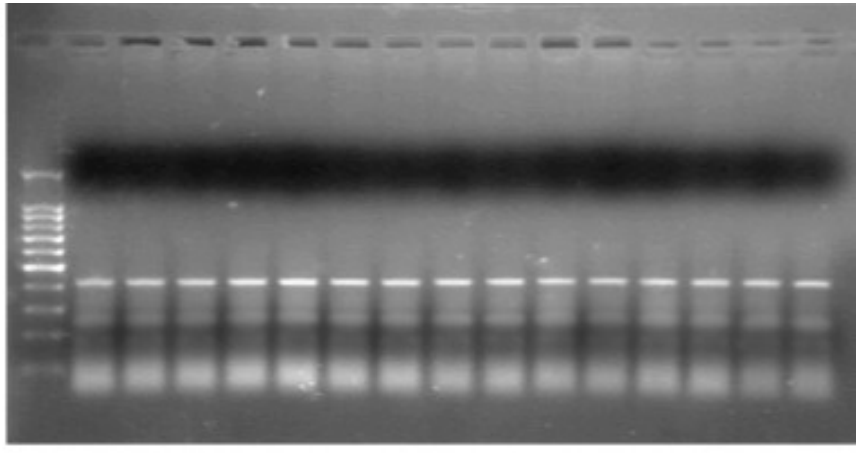

Sham Group

Control Group

Treatment Group

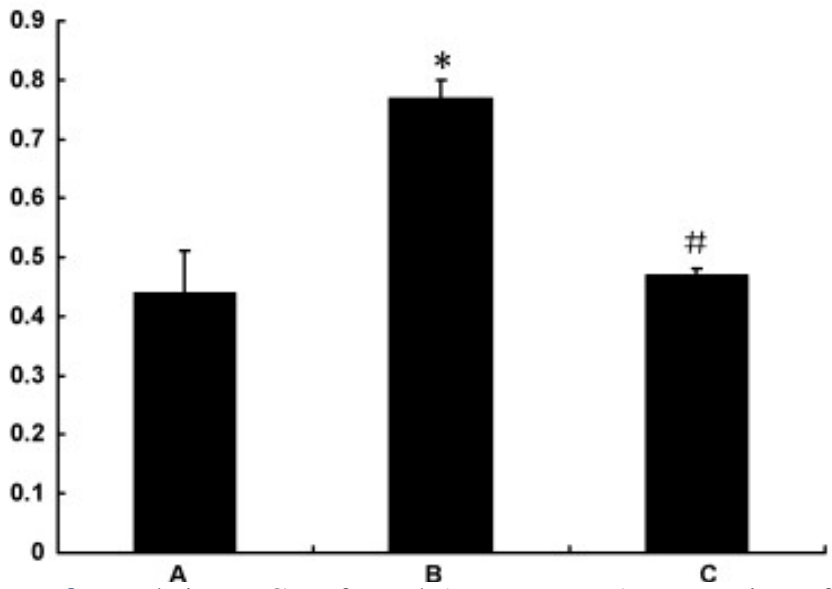

Figure 3: Real-time PCR of renal Ang II mRNA expression of representative rats in each group and semiquantitative analysis of the therapeutic effect of KQL on Ang II mRNA localization in the glomerulus and tubulointerstitium. Degree of glomerular and tubulointerstitial Ang II mRNA expression in Sham Group (A), 
Control Group (B) and Treatment Group (C). Each value represents mean \pm S.E.M. ${ }^{\#} P<0.05$, ${ }^{\#} P<0.001$ compared with Control Group; $* P<0.05,{ }^{* *} P<0.01$, compared with Sham Group.

(b)
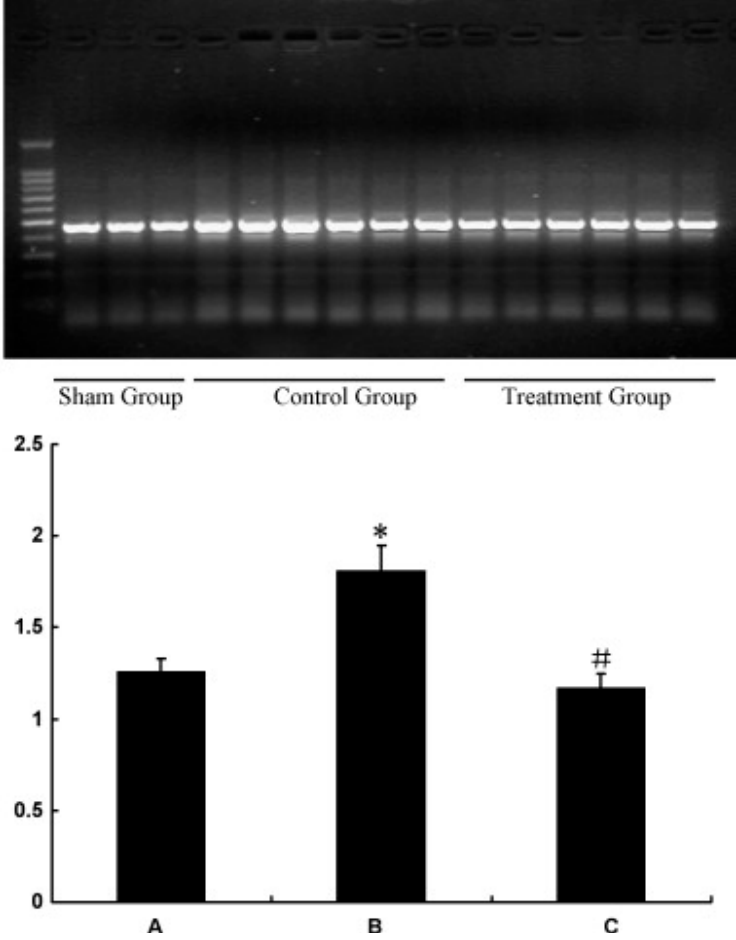

Figure 4: Real-time PCR of renal AT1R mRNA expression of representative rats in each group and semiquantitative analysis of the therapeutic effect of KQL on AT1RmRNA localization in the glomerulus and tubulointerstitium. Degree of glomerular and tubulointerstitial AT1RmRNA expression in Sham Group (A), Control Group (B) and Treatment Group (C). Each value represents mean \pm S.E.M. ${ }^{\#} P<0.05,{ }^{\# \#} P<0.001$ compared with Control Group; ${ }^{*} P<0.05,{ }^{* *} P<0.01$, compared with Sham Group.
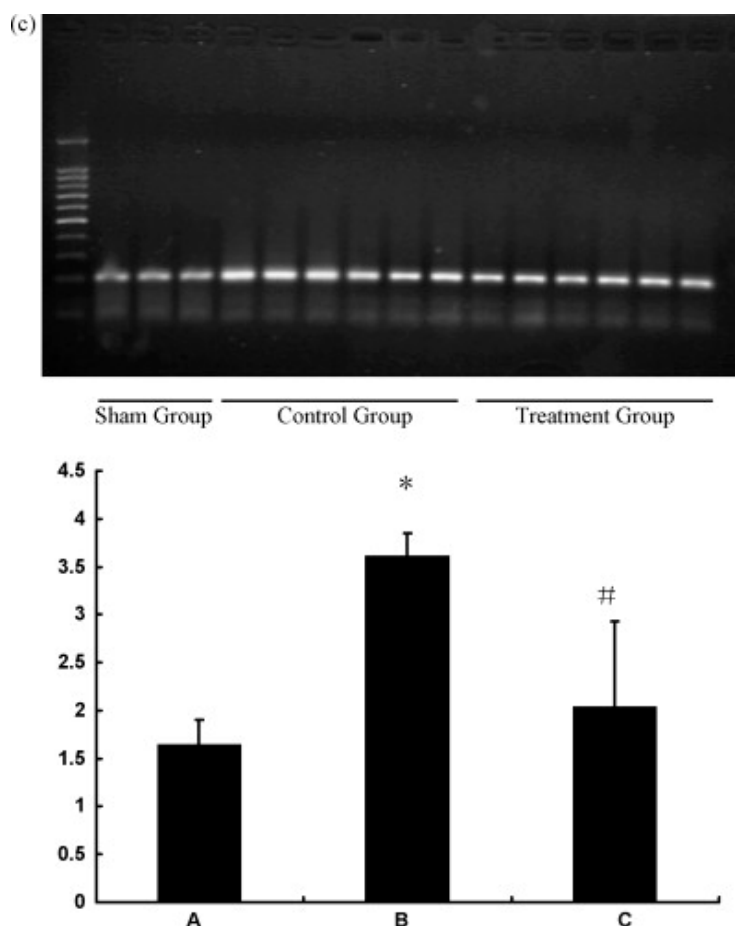
Figure 5: Real-time PCR of renal TNF- $\alpha$ mRNA expression of representative rats in each group and semiquantitative analysis of the therapeutic effect of KQL on TNF- $\alpha$ mRNA within the glomerulus and tubulointerstitium. Degree of glomerular and tubulointerstitial TNF- $\alpha$ mRNA expression in Sham Group (A), Control Group (B) and Treatment Group (C). Each value represents mean \pm S.E.M. ${ }^{\#} P<0.05,{ }^{\# \#} P<0.001$ compared with Control Group; *P $<0.05$, ${ }^{* *} P<0.01$, compared with Control Group.

\section{Effects of KQL on mean arterial blood pressure of uraemic rats}

After subtotal nephrectomy, hypertension developed in both Control Group and Treatment Group. Compared with Sham Group, blood pressure was significantly elevated from 2 to 8 weeks after nephrectomy ( $P<0.05$ or 0.01 ), and the rise in blood pressure was equivalent (systolic blood pressure 180-200 mmHg) in Control Group. After using KQL, hypertension was obviously suppressed in Treatment Group, showing average systolic blood pressure from 140 to $160 \mathrm{mmHg}$ (Table 2).

Table 2: Effect of KQL on systolic blood pressure (mmHg).

\begin{tabular}{lllllll}
\hline Group & $N$ & Before treatment & \multicolumn{2}{c}{ After treatment } & & \\
\cline { 3 - 7 } & & & Second week & Fourth week & Sixth week & Eighth week \\
\hline Sham & 12 & $137.31 \pm 14.72$ & $139.13 \pm 14.06$ & $125.50 \pm 7.15$ & $150.56 \pm 13.97$ & $129.63 \pm 29.16$ \\
Control & 12 & $140.50 \pm 23.55^{*}$ & $212.46 \pm 43.26$ & $199.92 \pm 23.55$ & $156.33 \pm 20.72$ & $202.44 \pm 15.09$ \\
Treatment & 12 & $141.77 \pm 26.45^{*}$ & $148.50 \pm 38.82^{\# \#}$ & $152.46 \pm 29.54^{\# \#}$ & $141.00 \pm 14.73^{\#}$ & $176.00 \pm 30.70^{\#}$ \\
\hline Values are means \pm S.E.M. & & & & \\
$* P<0.05$, compared with Sham Group. & & & & \\
$\# P<0.05$, compared with Control Group. & & & & \\
$\# \# P<0.01$, compared with Control Group. & &
\end{tabular}

\section{Effects of KQL on urine TGF- $\beta 1$ of uraemic rats}

High urine excretion of TGF- $\beta 1$ was expressed in both glomerular and tubulointerstitial injuries.

To further demonstrate the anti-inflammatory effect of the extract on rat chronic renal failure, the TGF- $\beta 1$ level was determined in the urine by ELISA. The results showed that compared with vehicle, KQL significantly reduced urinary TGF- $\beta 1$ levels, corrected by decrease level of Scr, throughout the entire disease course $(P<0.05)$, indicating that the treatment may primarily suppress the local immune and inflammatory response within the diseased kidney. In contrast, over expression of urine TGF- $\beta 1$ was found in control uraemic rats as compared with normal rats in Sham Group (Table 3). The experimental result showed the KQL administration significantly inversed high expression of urine TGF- $\beta$ in Nx rats, manifesting which attenuated the development of glomerular sclerosis.

Table 3: Effect of KQL on urine TGF- $\beta$ excretion.

\begin{tabular}{lll}
\hline Group & $\boldsymbol{N}$ & Urine TGF- $\boldsymbol{\beta}(\boldsymbol{\mu g} / \mathbf{L})$ \\
\hline Sham & 12 & $1.83 \pm 0.64$ \\
Control & 12 & $1.90 \pm 0.56^{*}$ \\
Treatment & 12 & $1.77 \pm 0.43^{\#}$ \\
\hline Values are means \pm S.E.M. \\
$* P<0.05$, compared with Sham Group. \\
$\# P<0.05$, compared with Control Group.
\end{tabular}

\section{Effects of $K Q L$ on localization of $N F-\kappa B$ in renal tissue}

Immunohistochemical analysis was performed to determine the localization of NF- $\kappa \mathrm{B}$ in the renal cortex (Fig. 1 and Fig. 2). NF- $\kappa B$, a critical transcriptional factor controlling inflammatory response, played a central role in inflammatory diseases, including kidney diseases (Muller et al., 2000). In normal rats, only tubular epithelial cells were weakly stained by the monoclonal anti$\mathrm{NF}-\kappa \mathrm{B}$ antibody, while glomeruli were hardly stained. In control Nx rats, however, proximal 
tubular epithelial cells, especially dilated tubules, were intensively stained by the anti-NF-kB antibody. In contrast, activation of the NF- $\kappa \mathrm{B}$ in tubular epithelial cells was not prominent in the KQL treated rats. The staining of NF- $\mathrm{BB}$ as shown in the control Nx rats increased NF- $\kappa \mathrm{B}-$ positive (intensively stained) area in the renal cortex, whereas the treated rats indicated markedly decreased NF- $\kappa B$-positive area. These data demonstrated that KQL markedly reduced the overload of NF- $\mathrm{KB}$ on the remnant tubular cells.

\section{Effects of KQL on mRNA levels of TNF- $\alpha$, Ang II and AT1R in renal tissue}

The effects of KQL on the gene expression of Ang II (Fig. 3), AT1R (Fig. 4) and TNF- $\alpha$ (Fig. 5) in the renal cortex were examined. We investigated the potential mechanisms whereby KQL suppressed rat tubular interstitial fibrosis and glomerular cirrhosis. In control Nx rats, there was a substantial increase in renal mRNA expression of TNF- $\alpha$, TNF- $\alpha$ inflammatory genes were significantly up-regulated by the treatment with KQL $(P<0.05)$. Furthermore, the mRNAs of Ang II and AT1R in renal cortex were attenuated by KQL compared with the control uraemic rats when they were administered after the establishment of nephrectomized. However, the renal mRNA levels of Ang II and AT1R were markedly increased in control Nx rats as compared with normal rats. The variation in the mRNA levels of TNF- $\alpha$, Ang II and AT1R in both treated and control uraemic rats are related to variation in the extent of CRF.

\section{DISCUSSION AND CONCLUSION}

The present study showed a protective action of KQL treatment in the chronic renal failure rats induced by $\mathrm{Nx}$, which promoted growth retardation, systemic arterial hypertension, impaired renal function, and severe albuminuria (Goncalves et al., 2004). These functional changes were accompanied by severe glomerulosclerosis, as well as expansion and intense macrophage infiltration of the interstitial area. Previous study indicated that these renal structural abnormalities, characteristics of the Nx and other models of progressive nephropathies, are a consequence of the concerted action of mechanical stress, caused by glomerular hypertension and hypertrophy, and inflammatory phenomena, comprising cell infiltration and/or proliferation and extracellular matrix accumulation (Fujihara et al., 2003). Moreover, a causal relationship appears to exist between these phenomena, because the distension of the glomerular walls due to intracapillary hypertension may trigger the local release of cytokines, growth factors, and, particularly, Ang II and AT1R (Lee et al., 1995).

We examined the renal function of rats including Scr, BUN and $24 \mathrm{~h}$ urine protein. Scr demonstrate the ability of kidney to remove creatinine from the blood and concentrate it in the urine. Renal dysfunction diminishes the ability to filter creatinine and so the Scr rises. Diseased or damaged kidneys cause an elevated BUN because the kidneys are less able to clear urea from the bloodstream. $24 \mathrm{~h}$ urine protein also reflected the function of kidney. Nx rats showed significant raise of Scr, BUN and 24 h urine protein, however, the apparently restored levels in Treatment Group indicating that KQL significant improve the kidney function of the Nx rat.

Hypertension is an important result in chronic renal disease and is associated with a more rapid rate of progression (Hannedouche et al., 1993). Therapeutic measures aiming to lower high blood pressure in progressive renal diseases have been widely used. Administration of KQL contributed a great beneficial effect against hypertension caused by the chronic renal failure. 
The production of extracellular matrix proteins in the interstitium is regulated and induced by growth factors derived from macrophages and tubular cells. In this process, one of the most relevant mediators is the TGF- $\beta$. KQL treatment group prevented the up-regulation of TGF- $\beta$ to the level of Sham control group, indicating that KQL showed protection against fibrogenic process.

Intrarenal Ang II distribution was profoundly changed after renal mass reduction (Noronha et al., 2002). In the process of renal disease progression another local mediator, Ang II, deserves consideration. Besides its hemodynamic effects, Ang II exerts actions on cells that contribute to inflammation and tissue fibrosis which favor the pathogenesis of renal disease. A preliminary report has suggested that, in the Nx model, Ang II is expressed in renal interstitial cells, paralleling the severity of renal injury (Noronha et al., 2002). In the present study, an increase in Ang II level was observed in control Nx rats, and was decreased in Treatment Group, indicating KQL may prevent the chronic renal failure by regulating local mediator, Ang II.

Renal expression of AT1R in rats appeared mostly in tubular cells, and to a lesser extent, at the interstitial area, whereas low expression was seen in vessels and glomeruli. This pattern was completely disrupted after $\mathrm{Nx}$, when dense AT1R expression could be demonstrated in interstitial cells, far exceeding in intensity the expression of AT1R in tubules. The exact meaning of this finding and the cell types involved are uncertain. The simultaneous presence at the interstitial area of large amounts of Ang II and AT1R may accelerate the progression of the nephropathy by a positive-feedback mechanism. Consistent with this view is the aggravation of the renal structural injury of $\mathrm{Nx}$, which was paralleled by the intensity of the inflammatory infiltration and of the interstitial expression of Ang II.

It has been thought that Ang II induces vascular fibrosis by stimulating TGF- $\beta$. Accordingly, TGF- $\beta$ induction is the initial step in promoting fibrosis during vascular remodeling in response to Ang II. Ang II and TGF- $\beta$ are critical mediators of renal fibrosis (Gaedeke et al., 2001). Ang II may mediate renal scarring through TGF- $\beta$ stimulation (Rumble et al., 1998 and Houlihan et al., 2002). In our study, the urinary TGF- $\beta$ excretion was in good accordance with the level of Ang II. At the same time, the increased TGF- $\beta$ level had been restored to the Sham group level in the KQL treatment group.

$\mathrm{NF}-\kappa \mathrm{B}$, a well accepted key transcriptional factor to regulate a variety of inflammatory responses (Barnes and Karin, 1997), is composed of p50 and p65 subunits, among which p65 is a potent transcriptional activator, strongly promoting inflammatory reaction in kidney diseases (Guijarro and Egido, 2001). In our study, using liquid extract isolated from clinical effective Chinese prescription, over expression activation of NF- $\mathrm{BB}$ was substantially suppressed. The result explained one of the mechanisms of KQL to improve renal function and correct high blood pressure.

Tumor necrosis factor- $\alpha$ is a potent pro-inflammatory cytokine which is produced by many cell types including monocytes/macrophages, and renal mesangial and epithelial cells. TNF- $\alpha$ may contribute to renal damage by inciting an inflammatory response within the kidney via induction of a variety of chemokines and adhesion molecules (Tipping et al., 1999). The TNF- $\alpha$ expressions within the glomerulus and tubulointerstitium were significantly different in the KQL 
treatment group compared with the control group, which may due to the anti-inflammatory function of KQL through preventing the expression of TNF- $\alpha$.

On the basis of the present study, the efficacy of KQL was likely due to the simultaneous blockade of the hemodynamic and pro-inflammatory actions of Ang II, AT1R and its derivatives as TNF- $\alpha$, NF- $\kappa$ B, TGF- $\beta$ and by abrogation of the complex interplay between hypertension and inflammation. It is noteworthy that KQL afforded partial regression of the chronic renal failure induced by Nx model. This observation suggests that continued stimulation of Ang II, AT1 receptors and production of inflammatory factors played an important pathogenic role even during the late phases of the process, KQL was a vigorous and persistent treatment to prevent further renal deterioration.

In conclusion, KQL administration ameliorates renal dysfunction, renal fibrosis and downregulate many critical anti-inflammatory factors in rats with $\mathrm{Nx}$-induced chronic renal failure. Taken together with our previous works (He et al., 2003 and He and Cai, 2005a) and the present results, it is likely that KQL-induced reduction of renal rennin-angiotensin system is mediated, at least partly, by reducing the overload of inflammatory factors activity on remnant kidney unit. In summary, KQL partially reversed the nephropathy and renal inflammation associated with the Nx model. Clinical mechanism studies are further needed to establish how this scheme may eventually become a new weapon in the limited arsenal available to attenuate or prevent human progressive chronic renal failure.

$\begin{array}{ll}\text { ABBREVIATIONS } & \\ \text { KQL } & \text { Kangqianling decoction } \\ \text { Scr } & \text { serum creatinine } \\ \text { BUN } & \text { blood urea nitrogen } \\ \text { Nx } & 5 / 6 \text { renal ablation } \\ \text { TGF- } \beta 1 & \text { transforming growth factor- } \beta 1 \\ \text { Ang II } & \text { angiotensin II } \\ \text { TNF- } \alpha & \text { tumor necrosis factor- } \alpha \\ \text { NF-Kb } & \text { nuclear factor- } \kappa \text { B } \\ \text { AT1R } & \text { angiotensinII-receptor }\end{array}$

ACKNOWLEDGEMENTS

This work was financially supported by the National Basic Research Program (973 program) of China (2004CB518605), project of E-institutes of Shanghai Municipal Education Commission (E03008), the Creative Team Program of Science and Technology Commission of Shanghai Municipality (06DZ05906) and the Program of National Natural Science Fund (30572286).

REFERENCES

Allison, A.E., 2000. Molecular basis of renal fibrosis. Pediatric Nephrology 15, 290-301.

Barnes, P.J., Karin, M., 1997. Nuclear factor-kappaB: a pivotal transcription factor in chronic inflammatory diseases. New England Journal of Medicine 336, 1066-1071. 
Chen, Z.P., He, L.Q., 2006. Clinical study on the effects of “Kangqianling Granule” on bone metabolism in chronic renal failure patients. Journal of Shanghai TCM 40, 34-35.

Fujihara, C.K., Antunes, G.R., Mattar, A.L., Andreoli, N., Malheiros, D.M., Noronha, I.L., Zatz, R., 2003. Cyclooxygenase-2 (COX-2) inhibition limits abnormal COX-2 expression and progressive injury in the remnant kidney. Kidney International 64, 2172-2181.

Gaedeke, J., Peters, H., Noble, N.A., Border, W.A., 2001. Angiotensin II, TGF-beta and renal fibrosis. Contribution Nephrology 135, 153-160.

Goncalves, A.R.R., Fujihara, C.K., Mattar, A.L., Malheiros, D.M.A.C., Noronha, I.D.L., De Nucci, G., Zatz, R., 2004. Renal expression of COX-2, ANG II, and AT1 receptor in remnant kidney: strong renoprotection by therapy with losartan and a nonsteroidal antiinflammatory. American Journal of Physiology-Renal Physiology 286, F945-F954.

Guijarro, C., Egido, J., 2001. Transcription factor-kappa B (NF-kappa B) and renal disease. Kidney International 59, 415-424.

Guo, Q.Y., 2006. Curative effect observation of hypertension nephropathywith modified Shengqidihuang decoction and Taohongsiwu decoction. Journal of Henan University of Chinese Medicine 21, 28-32.

Hannedouche, T., Chauveau, P., Kalou, F., Albouze, G., Lacour, B., Jungers, P., 1993. Factors affecting progression in advanced chronic renal failure. Clinical Nephrology 39, 312320.

He, L.Q., Cai, G., 2005a. The clinical observation of the JIAN-PI-QIN-HUI prescription on spleen deficiency anddampness heat style patientswith chronic renal failure. Journal of International Medical Science of China 14, 270-274.

He, L.Q., Li, J., Li, Y., 2005b. The effect of "FUZHENGHUOXUEdecoction” on the expressions of fibronectin and transforming growth factor-_mRNAin renal tissue of the CRF rats. Journal of Chinese Medicine 46, 454-457.

He, L.Q., Wang, Y., Cao, H.X., Li, J., 2003. The effect of kangQianling decoction on PDGFmRNA, TNF_-mRNA expression of CRF rat renal tissue. Journal of China Experiments the Square to Learn 9, 29-32.

Houlihan, C.A., Akdeniz, A., Tsalamandris, C., Cooper, M.E., Jerums, G., Gilbert, R.E., 2002. Urinary transforming growth factor-beta excretion in patients with hypertension, type 2 diabetes, and elevated albumin excretion rate: effects of angiotensin receptor blockade and sodium restriction. Diabetes Care 25, 1072-1077.

Jin, Y.M., Zhu, R., Zhang, C.S., Li, M., Deng, Y.Y., Shen, L.M., 2006. Treating hematomas after renal puncture with "Taohong Siwu decoction”. Journal of Shanghai Traditional Chinese Medicine 40, 30-32. 
Karkar, A.M., Koshino, Y., Cashman, S.J., Dash, A.C., Bonnefoy, J., Meager, A., Rees, A.J., 1992. Passive immunization against TNF alpha and IL-1B protects from LPS enhancing glomerular injury in nephrotoxic nephritis in rats. Clinical and Experimental Immunology 90, 312-318.

Lee, L.K., Meyer, T.W., Pollock, A.S., Lovett, D.H., 1995. Endothelial cell injury initiates glomerular sclerosis in the rat remnant kidney. Journal of Clinical Investigation 96, 953964.

Li, J., He, L.Q., Li, Y., Hou, W.G., 2003. The effect of kang qian ling 2 decoction on serum lipidmetabolism of chronic renal fail rats. Journal of InternationalMedical Science of China 3, 204-206.

Muller, D.N., Dechend, R., Mervaala, E.M., Park, J.K., Schmidt, F., Fiebeler, A., Theuer, J., Breu, V., Ganten, D., Haller, H., Luft, F.C., 2000. NF-_B inhibition ameliorates angiotensin II-induced inflammatory damage in rats. Hypertension 35, 193-201.

Ng, Y.Y., Huang, T.P., Yang,W.C., Chen, Z.P., Yang, A.H., Mu,W., Nikolic-Paterson, D.J., Atkins, R.C., Lan, H.Y., 1998. Tubular epithelial-myofibroblast transdifferentiation in progressive tubulointerstitial fibrosis in 5/6 nephrectomized rats. Kidney International 54, 864-876.

Noronha, I.L., Fujihara, C.K., Zatz, R., 2002. The inflammatory component in progressive renal disease-are interventions possible (Abstract)? Nephrology Dial Transplant 17, 363.

Ruiz-Ortega, M., Egido, J., 1997. Angiotensin II modulates cell growthrelated events and synthesis of matrix proteins in renal interstitial fibroblasts. Kidney International 52, 1497-1510.

Rumble, J.R., Gilbert, R.E., Cox, A.,Wu, L., Cooper,M.E., 1998. Angiotensin converting enzyme inhibition reduces the expression of transforming growth factor-beta(1) and type IV collagen in diabetic vasculopathy. Journal of Hypertension 16, 1603-1609.

Tipping, P.G., Kitching, A.R., Cunningham, M.A., Holdsworth, S.R., 1999. Immunopathogenesis of crescentic glomerulonephritis. Current Opinion Nephrology Hypertension 8, 281-286.

Weistuch, J.M., Dworkin, L.D., 1992. Does essential hypertension cause end-stage renal disease? Kidney International 41, S33-S37.

Wolf, G., Ziyadeh, F.N., Stahl, R.A., 1999. Angiotensin II stimulates expression of transforming growth factor beta receptor type II in cultured mouse proximal tubular cells. Journal of Molecular Medicine 77, 556-564. 
Xia, Y.B., Lu, G.Y., Xiong, P.H., Xie, Y., Shen, L., 2007. Plasma concentration changes and the clinical significance of plasminogen activator, plasminogen activator receptor and plasminogen activator inhibitor-1 in patients with chronical glomerulonephritic and the intervenient research on them. Journal of Suzhou University 27, 105-107. 